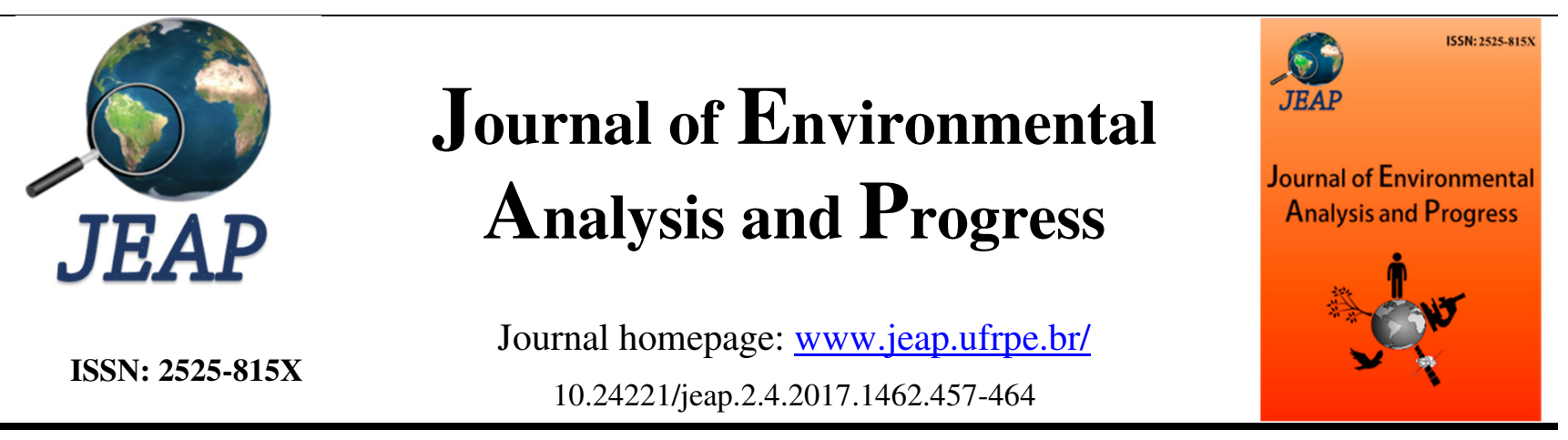

\title{
Precipitação esperada, em diferentes níveis de probabilidade, na região de Juazeiro-BA
}

\section{Expected rainfall at different levels of probability in the region of Juazeiro-BA}

Edgo Jackson Pinto Santiago a , Gertrudes Macário de Oliveiraa , Mário de Miranda Vilas Boas Ramos Leitão ${ }^{\mathrm{b}}$, Magna Soelma Beserra de Moura $^{\mathrm{c}}$, Iris Santana Gonçalves ${ }^{\mathrm{a}}$

a Universidade do Estado da Bahia-UNEB, Departamento de Tecnologia e Ciências Sociais-DTCS, Programa de PósGraduação em Horticultura Irrigada-PPGHI, Campus III, Juazeiro-BA, Brasil. Av. Edgard Chastinet, SN, São Geraldo, Juazeiro, BA, Brasil. CEP: 48900-000. E-mail: edgoj@hotmail.com; gemoliveira@uneb.br; irissg@hotmail.com.

b Universidade Federal do Vale do São Francisco-UNIVASF, Av. Antônio Carlos Magalhães, n. 510, Country Club, Juazeiro, BA, Brasil. CEP: 48902-300. E-mail: mario.miranda@univasf.edu.br.

c EMBRAPA Semiárido, Rodovia BR-428, Km 152, s/n, Zona Rural, Petrolina-PE, Brasil. CEP: 56302-970. E-mail: magna_upa@hotmail.com.

A R T I C L E I N F O

Recebido 30 Jun 2017

Aceito 03 Ago 2017

Publicado 13 Out 2017

\begin{abstract}
A B S T R A C T
Rainfall events are preponderant for the development of crops dependent on its occurrence. Given that climate predictions are not always accurate, techniques that can help predictions, such as rainfall estimates, based on probability levels of their occurrence, can be a suitable alternative. The study aimed to determine the expected rainfall, at different probability levels, for the region of Juazeiro, State of Bahia, as well as the period of lower risk of losses in the harvesting of fine grapes due to the occurrence of rainfall. The study was carried out with daily rainfall data from a series of 40 years, obtained from the Agrometeorological station of Mandacaru in the municipality of Juazeiro, Bahia. The gamma distribution function obtained monthly estimates of likely rainfall for the $90,80,75,60,50,45,40,35$, and $30 \%$ levels of probability. The Kolmogorov-Smirnov adhesion test was applied at the $5 \%$ level of significance to verify the fit of the data. The range distribution presented a good fit to estimate the probable precipitation at different probability levels in the region of Juazeiro-BA. The average values of monthly rainfall occurred near the $40 \%$ probability level. The most suitable time for the cultivation of rainfed in the region was the February and March each 2-months. The lower probabilities of rainfall from May to October provide a lower risk of losses in the harvesting of fine grapes in irrigated crops, in the region of Juazeiro, BA.
\end{abstract}

Keywords: Rainfall, semiarid, range distribution.

\section{R E S U M O}

Eventos de chuvas são preponderantes para o desenvolvimento dos cultivos agrícolas totalmente dependentes de sua ocorrência. Tendo em vista que previsões climáticas nem sempre são precisas, técnicas que possam auxiliar nas previsões, como estimativas de precipitações, com base em níveis de probabilidade de sua ocorrência, podem ser uma alternativa adequada. $\mathrm{O}$ estudo objetivou determinar a precipitação pluviométrica esperada, em diferentes níveis de probabilidade, para a região de Juazeiro, BA bem como a época de menor risco de perdas na colheita de uvas finas por causa da ocorrência de chuvas. O estudo foi realizado com dados dos registros pluviométricos diários de uma série de 40 anos, obtidos junto à estação Agrometeorológica de Mandacaru no município de Juazeiro-BA. As estimativas 
mensais da precipitação pluviométrica provável para os níveis de 90, 80, 75, 60, 50, $45,40,35$ e $30 \%$ de probabilidade foram obtidas pela função de distribuição gama. Aplicou-se o teste de aderência de Kolmogorov-Smirnov, ao nível de 5\% de significância para verificar o ajuste dos dados. A distribuição gama apresentou bom ajuste para estimar a precipitação provável em diferentes níveis de probabilidade na região de Juazeiro, BA. Os valores médios de precipitação pluvial mensal ocorreram próximo ao nível de $40 \%$ de probabilidade. A época mais adequada para o cultivo de sequeiro na região foi o bimestre fevereiro e março. As menores probabilidades de ocorrência de chuva de maio a outubro proporcionam menor risco de perdas na colheita de uvas finas em cultivo irrigado, na região de Juazeiro, BA.

Palavras-Chave: Chuva, semiárido, distribuição gama.

\section{Introdução}

O município de Juazeiro-BA situa-se no extremo norte do estado, às margens do rio São Francisco; destaca-se no cenário nacional pela prática da agricultura irrigada. Porém, apresenta extensa área onde se realiza a agropecuária dependente de chuvas. Tem como características climáticas, acentuada irregularidade da precipitação, elevadas temperaturas e baixa umidade do ar na maior parte do ano, grande déficit hídrico e elevadas taxas de evapotranspiração. Em regiões onde os recursos hídricos são escassos, a água pluvial é responsável por grande parte do desenvolvimento agrário. Portanto, a ocorrência de chuva é a forma menos dispendiosa e ambientalmente mais adequada para a utilização de água na agricultura (Vieira et al., 2010).

As quantidades alusivas de precipitações representadas pelos seus volumes, bem como os regimes sazonais ou diários, a distribuição temporal e as intensidades de chuvas individuais, são aspectos que interferem nas condições do ambiente (Sousa et al., 2006). Para Vela et al. (2007), o conhecimento do histórico da precipitação de determinada região é de suma importância para o monitoramento dos impactos causados pelo excesso ou falta prolongada de precipitação. Todavia, as chuvas irregulares variam espacialmente de um ano para outro, não obstante a existência de modelos hidrológicos para estimar a precipitação pluvial, várias metodologias têm sido utilizadas para se analisar a variabilidade da precipitação pluvial melhorando o controle no monitoramento dos recursos hídricos (Balme et al., 2006).

A estimativa da chuva com determinado nível de probabilidade é de grande importância no planejamento agrícola, possibilitando determinar a melhor época de preparo do solo, colheita, semeadura, aplicação de adubos, defensivos, e lâmina suplementar de irrigação (Ávila et al., 2009). Barreto et al. (2015) relatam que o uso de funções de probabilidade está diretamente ligado à natureza dos dados a que ela se relaciona; sendo possível estimar através de séries de dados pluviométricos, os cálculos de probabilidade de ocorrência de precipitações, ferramenta importante para o planejamento de operações agrícolas e humanas. Neste contexto, este estudo objetivou determinar a precipitação pluviométrica esperada, com diferentes níveis de probabilidade de ocorrência e época com menor risco de perdas na colheita de uvas finas em função da ocorrência de chuvas.

\section{Material e Métodos}

O estudo foi realizado com dados pluviométricos diários de uma série de 40 anos (1975 a 2014), obtidos na Estação Agrometeorológica de Mandacaru $\left(09^{\circ} 24^{\prime} \mathrm{S}\right.$ e $40^{\circ} 26^{\prime} \mathrm{W}$ ), localizada no município de JuazeiroBA.

As estimativas de precipitação pluviométrica mensal provável foram obtidas para os níveis de $90,80,75,60,50,45,40,35$ e $30 \%$ de probabilidade, utilizando-se a função de distribuição gama (Assis et al., 1996). A função densidade de probabilidade da função gama é representada pela Eq. 1:

$$
\begin{aligned}
& \mathrm{f}(\mathrm{x})=\frac{1}{\beta \alpha \cdot \tau(\alpha)} \mathrm{x}^{\alpha-1} \mathrm{e}^{-\frac{\mathrm{x}}{\beta}}, \text { se } 0<\mathrm{x}<\infty \\
& \text { ou } \\
& \mathrm{f}(\mathrm{x})=0, \text { se }-\infty<\mathrm{x}<0
\end{aligned}
$$

onde $\tau(\alpha)$ é a função gama; $\alpha$ é o parâmetro de forma (adimensional); $\beta$ é o parâmetro de escala $(\mathrm{mm}) ; e$ é a base do logaritmo neperiano; $\mathrm{x}$ é o total de precipitação $(\mathrm{mm})$.

A função gama $(\tau(\alpha))$ é dada pela Eq. $2 \mathrm{e}$ os parâmetros $\alpha$ e $\beta$ da distribuição gama foram determinados pelo método de máxima verossimilhança (Assis et al., 1996), conforme as Eq. 3, 4 e 5, respectivamente.

$\tau(x)=\int_{0}^{\infty} e^{-x} x^{\alpha-1} d x, \quad \alpha>0$ 
$\alpha=\frac{1}{4 \mathrm{~A}}\left(1+\sqrt{1+\frac{4 \mathrm{~A}}{3}}\right)$

$\beta=\frac{X_{a}}{\alpha}$

$A=\ln X_{a}-X_{g}$

onde $X_{a}$ e $X_{g}$, a média aritmética e a média geométrica, respectivamente, das observações em escala mensal.

Como a série de dados continha valores nulos, não foi possível utilizar distribuição cumulativa gama, pois nesse caso não é possível estima os parâmetros $(\alpha$ e $\beta)$ pelo método de máxima verossimilhança. Utilizou-se para estimativa da frequência de ocorrência de eventos de precipitação, a distribuição cumulativa gama mista, determinada segundo Assis et al. (1996) e Thom (1951), pelas Eqs. 6 e 7, respectivamente:

$$
\begin{aligned}
& F(x)=P_{0}+\left(1-P_{0}\right) \cdot G(x) \\
& P_{0}=\frac{N_{0}}{N}
\end{aligned}
$$

onde $\mathrm{P}_{0}$ é a probabilidade de ocorrência de valores nulos; $\mathrm{G}(\mathrm{x})$ é a função cumulativa gama de probabilidade; $\mathrm{N}_{0}$ é o número de valores nulos da série; $\mathrm{N}$ é o tamanho da série.

Para as estimativas dos valores de precipitação provável utilizaram-se as funções INV.GAMA e DIST.GAMA, que retornam, respectivamente, o valor de precipitação associado a um determinado nível de probabilidade, e o valor da probabilidade associado a um dado valor de precipitação. Ambas as funções levam em consideração os valores de $\alpha$ e $\beta$.

$\mathrm{Na}$ análise dos totais de precipitação de cada período, para verificar a porcentagem de ocorrência, foram consideradas quatro classes de chuvas acumuladas mensais, a fim de obter-se adequado grau de precisão nas estimativas de probabilidades de ocorrência para os períodos secos e chuvosos (Andrade et al., 2009). Considerou-se como mês seco (S), precipitação acumulada de 0 a $50 \mathrm{~mm}$; mês pouco chuvoso (PC), de 50 a $100 \mathrm{~mm}$; mês chuvoso (C), de 100 a $200 \mathrm{~mm}$; e mês bastante chuvoso (BC), precipitação acima de $200 \mathrm{~mm}$. As precipitações inferiores a $0,5 \mathrm{~mm}$ foram consideradas como nulas e como $1,0 \mathrm{~mm}$ as do intervalo de $0,5 \mathrm{~mm}$ a $1,0 \mathrm{~mm}$.

As probabilidades de períodos secos $\mathrm{P}(\mathrm{S})$, pouco chuvoso $\mathrm{P}(\mathrm{PC})$, chuvoso $\mathrm{P}(\mathrm{C})$ e bastante chuvoso $\mathrm{P}(\mathrm{BC})$ foram calculadas conforme as Eqs. 8 a 11. As probabilidades condicionais de: períodos secos, dado que o período anterior também foi seco $\mathrm{P}(\mathrm{S} / \mathrm{S})$; período seco, dado que o período anterior foi chuvoso $\mathrm{P}(\mathrm{S} / \mathrm{C})$; período chuvoso, dado que o período anterior foi chuvoso $\mathrm{P}(\mathrm{C} / \mathrm{C})$; e período chuvoso, dado que o período anterior foi seco $\mathrm{P}(\mathrm{C} / \mathrm{S})$, calculadas conforme as Eqs. de 12 a 15. Vale salientar que, para o cálculo das probabilidades condicionais, foram consideradas apenas duas classes de chuvas: período seco (S) de 0 a $50 \mathrm{~mm}$ e período chuvoso (C) acima de $50 \mathrm{~mm}$.

$$
\begin{aligned}
& P(S)=\frac{F S}{F S+F P C+F C+F B C} \\
& P(P C)=\frac{F P C}{F S+F P C+F C+F B C} \\
& P(C)=\frac{F C}{F S+F P C+F C+F B C}
\end{aligned}
$$

$$
P(B C)=\frac{F B C}{F S+F P C+F C+F B C}
$$

$$
\begin{aligned}
& \mathrm{P}(\mathrm{S} / \mathrm{S})=\frac{\mathrm{FSS}}{\mathrm{FS}} \\
& P(S / C)=1-P(C / C) \\
& P(C / C)=\frac{F C C}{F C} \\
& P(C / S)=1-P(S / S)
\end{aligned}
$$

onde FS = frequência de períodos secos; FPC = frequência de períodos pouco chuvosos; $\mathrm{FC}=$ frequência de períodos chuvosos; $\mathrm{FBC}=$ frequência de períodos bastante chuvosos; FSS = frequência de períodos secos precedidos de períodos secos; $\mathrm{FSC}=$ frequência de períodos secos precedidos de períodos chuvosos; e FCC = frequência de períodos chuvosos precedidos de períodos chuvosos.

Para avaliação do ajuste associada aos valores de frequências de precipitações observados com os estimados, nos vários níveis de probabilidade, pela distribuição de probabilidade gama, aplicou-se o teste de aderência de Kolmogorov-Smirnov, ao nível de $5 \%$ de significância.

\section{Resultados}

Com base na série de dados de precipitação pluviométrica, observou-se, para o 
município de Juazeiro-BA, que o período chuvoso tem início em novembro e se estende até abril, podendo ocorrer chuvas a partir do mês de outubro. Os menores índices pluviométricos ocorrem nos meses de junho a setembro.

Constatou-se que durante todo o período de estudo, a função de distribuição gama, segundo o teste de aderência de Kolmogorov-Smirnov a $5 \%$ de probabilidade, ajustou-se bem às quatro classes de chuvas acumuladas mensais estudadas, conforme mostra a Figura 1. Nela são apresentados, para as quatro classes de chuvas acumuladas mensais, valores da distribuição de frequência de ocorrência observadas e prováveis, bem como o comportamento assimétrico dos eventos de precipitações, representados pela função densidade de probabilidade da distribuição gama.
A

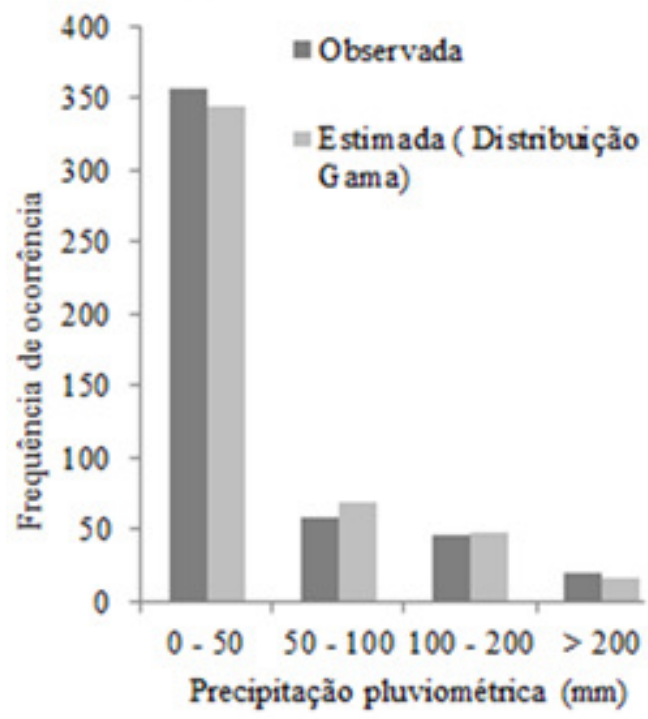

B

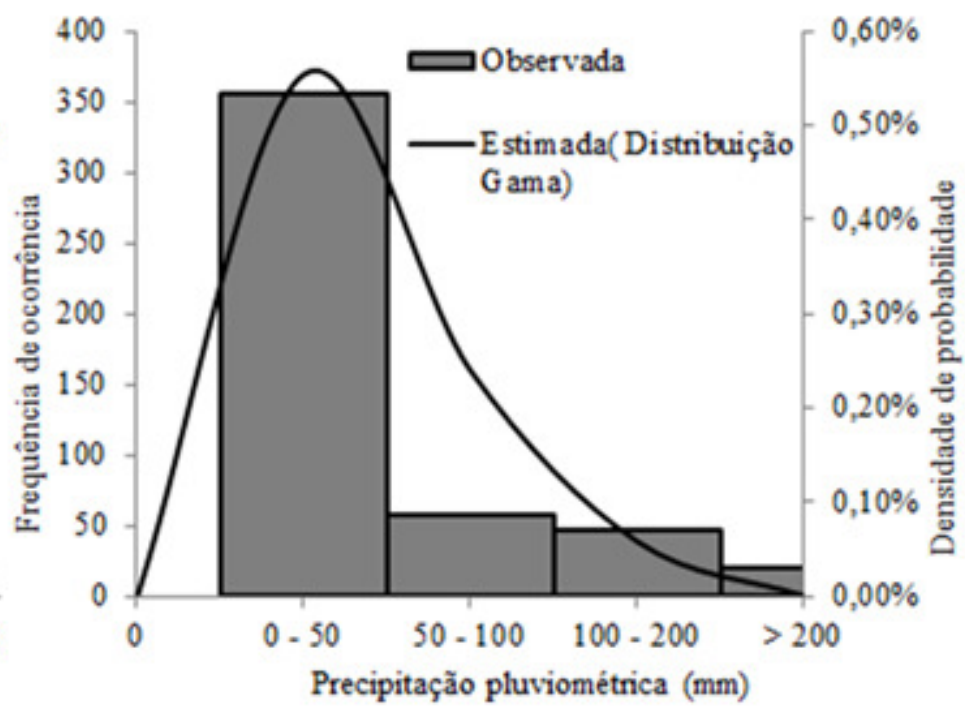

Figura 1. Distribuição de frequência da ocorrência de chuvas para quatro classes acumuladas mensais: frequência da ocorrência pluviométrica mensal observada e estimada pela distribuição gama (A), histograma de frequência de precipitação mensal observada sobreposta pela função densidade de probabilidade gama, no município de Juazeiro-BA, período de 1975 a 2014.

Na Figura 1A observa-se um decréscimo das frequências de ocorrências de precipitações mensais prováveis, estimada pela distribuição gama, no intervalo de 0 a $50 \mathrm{~mm}$, comparado aos valores observados, seguido de um pequeno aumento na estimativa de ocorrência prováveis no intervalo de 50 a $100 \mathrm{~mm}$. Para os intervalos de 100 a $200 \mathrm{~mm}$, e acima de $200 \mathrm{~mm}$, foram observadas pequenas diferenças entre os valores estimados e observados. O número de ocorrências de precipitações mensais superiores a $200 \mathrm{~mm}$, em toda a série histórica, correspondeu a menor frequência de observações, representando, ao todo, 20 eventos, significando apenas $4,16 \%$ da série de dados.

$\mathrm{Na}$ Tabela 1 são apresentados os parâmetros de forma $(\alpha)$ e escala $(\beta)$ da distribuição gama, os dados de precipitação média mensal para o período de estudo e as estimativas das precipitações mensais prováveis em diferentes níveis de probabilidade.

Tabela 1. Estimativas dos parâmetros da distribuição Gama $(\alpha, \beta)$, da precipitação média mensal (mm) e precipitação mensal média provável $(\mathrm{mm})$ para diferentes níveis de probabilidade, em Juazeiro-BA. Série histórica: 1975 a 2014.

\begin{tabular}{|c|c|c|c|c|c|c|c|c|c|c|c|c|c|}
\hline \multirow{3}{*}{ Mês } & \multicolumn{13}{|c|}{ Precipitação pluviométrica $(\mathbf{m m})$} \\
\hline & \multirow{2}{*}{$\alpha$} & \multirow{2}{*}{$\beta$} & \multirow[t]{2}{*}{ Média } & \multicolumn{10}{|c|}{ Nível de probabilidade (\%) - P $(X \geq x i)$} \\
\hline & & & & 90 & 80 & 75 & 70 & 60 & 50 & 45 & 40 & 35 & 30 \\
\hline Jan & 0,74 & 120,68 & 88,9 & 4,8 & 12,8 & 17,8 & 23,3 & 36,6 & 53,3 & 63,4 & 74,8 & 88,1 & 103,7 \\
\hline Fev & 1,09 & 79,11 & 84,3 & 10,4 & 20,9 & 26,5 & 32,3 & 45,3 & 60,4 & 70,8 & 80,7 & 91,8 & 104,6 \\
\hline Mar & 1,13 & 108,05 & 118,6 & 15,4 & 30,3 & 38,3 & 46,6 & 64,8 & 85,9 & 100,5 & 114,2 & 129,7 & 147,5 \\
\hline Abr & 1,14 & 53,13 & 57,3 & 7,5 & 14,7 & 18,4 & 22,4 & 31,0 & 41,0 & 50,4 & 57,2 & 64,9 & 73,7 \\
\hline
\end{tabular}


Journal of Environmental Analysis and Progress V. 02 N. 04 (2017) 457-464

\begin{tabular}{cccccccccccccc} 
Mai & 0,82 & 27,96 & 19,4 & 1,5 & 3,5 & 4,6 & 5,9 & 8,8 & 12,5 & 17,0 & 19,9 & 23,2 & 27,0 \\
Jun & 1,00 & 9,71 & 7,8 & 1,0 & 1,9 & 2,4 & 3,0 & 4,2 & 5,6 & 7,8 & 8,9 & 10,2 & 11,7 \\
Jul & 1,11 & 6,42 & 5,6 & 0,9 & 1,6 & 2,0 & 2,4 & 3,2 & 4,2 & 5,9 & 6,7 & 7,6 & 8,7 \\
Ago & 3,82 & 1,00 & 2,0 & 1,3 & 1,6 & 1,8 & 1,9 & 2,1 & 2,4 & 3,7 & 4,0 & 4,3 & 4,6 \\
Set & 1,08 & 5,37 & 2,9 & 0,9 & 1,2 & 1,4 & 1,6 & 2,0 & 2,6 & 4,7 & 5,4 & 6,2 & 7,0 \\
Out & 0,86 & 19,27 & 7,0 & 1,1 & 1,9 & 2,3 & 2,7 & 3,8 & 5,1 & 12,5 & 14,6 & 16,9 & 19,6 \\
Nov & 1,30 & 42,59 & 47,1 & 7,7 & 14,0 & 17,3 & 20,6 & 27,7 & 35,8 & 47,3 & 53,3 & 59,9 & 67,4 \\
Dez & 1,08 & 73,19 & 76,2 & 9,1 & 18,3 & 23,2 & 28,4 & 40,0 & 53,4 & 64,3 & 73,4 & 83,6 & 95,4 \\
\hline
\end{tabular}

Observa-se que, de modo geral, os valores médios de precipitação pluvial mensal ocorreram próximo ao nível de $40 \%$ de probabilidade (Tabela 1). Tal fato sugere que, a apropriação daqueles valores para utilização no planejamento de sistema de irrigação pode comprometer sua utilização, por não representar a lâmina mínima precipitável na região, logo, ao ser levada em consideração, resulta em subutilização de equipamentos e acessórios.
Constatou-se que o parâmetro $\alpha$ variou de 0,74 em janeiro a 3,82 em agosto, não excedendo o valor de 100 em nenhum outro mês (Tabela 1). Isso mostra que os dados mensais de chuva de toda série histórica foram bem ajustados pela distribuição gama.

Os resultados encontrados através da análise probabilística da ocorrência de períodos secos, pouco chuvosos, chuvosos, bastante chuvosos, bem como suas probabilidades condicionais, são apresentados na Tabela 2.

Tabela 2. Probabilidade de ocorrência de precipitações de períodos secos $\mathrm{P}(\mathrm{S})$, pouco chuvosos $\mathrm{P}(\mathrm{PC})$, chuvosos $\mathrm{P}(\mathrm{C})$, bastante chuvoso $\mathrm{P}(\mathrm{BC})$, períodos secos, dado que o período anterior foi seco $\mathrm{P}(\mathrm{S} / \mathrm{S})$, período chuvoso, dado que o período anterior foi seco $\mathrm{P}(\mathrm{C} / \mathrm{S})$, período chuvoso, dado que o período anterior foi chuvoso $\mathrm{P}(\mathrm{C} / \mathrm{C})$ e período seco, dado que o período anterior foi chuvoso $\mathrm{P}(\mathrm{S} / \mathrm{C})$, em Juazeiro-BA. Período: 1975 a 2014.

\begin{tabular}{|c|c|c|c|c|c|c|c|c|}
\hline \multirow{2}{*}{ Mês } & \multicolumn{8}{|c|}{ Probabilidade de ocorrência de precipitações (\%) } \\
\hline & $\mathbf{P}(\mathbf{S})$ & $\mathbf{P}(\mathbf{P C})$ & $\mathbf{P}(\mathbf{C})$ & $\mathbf{P}(\mathbf{B C})$ & $\mathbf{P}(\mathbf{S} / \mathbf{S})$ & $\mathbf{P}(\mathbf{C} / \mathbf{S})$ & $\mathbf{P}(\mathbf{C} / \mathrm{C})$ & $\mathbf{P}(\mathbf{S} / \mathbf{C})$ \\
\hline Jan & 56,40 & 20,50 & 12,80 & 10,30 & 59,10 & 40,90 & 55,60 & 44,40 \\
\hline $\mathrm{Fev}$ & 45,00 & 20,00 & 27,50 & 7,50 & 50,00 & 50,00 & 58,80 & 41,20 \\
\hline Mar & 32,50 & 22,50 & 20,00 & 25,00 & 33,30 & 66,70 & 68,20 & 31,80 \\
\hline Abr & 52,50 & 35,00 & 10,00 & 2,50 & 76,90 & 23,10 & 59,30 & 40,70 \\
\hline Mai & 95,00 & 2,50 & 2,50 & 0,00 & 95,20 & 4,80 & 5,30 & 94,70 \\
\hline Jun & 100,00 & 0,00 & 0,00 & 0,00 & 100,00 & 0,00 & 0,00 & 100,00 \\
\hline Jul & 100,00 & 0,00 & 0,00 & 0,00 & 100,00 & 0,00 & - & - \\
\hline Ago & 100,00 & 0,00 & 0,00 & 0,00 & 100,00 & 0,00 & - & - \\
\hline Set & 100,00 & 0,00 & 0,00 & 0,00 & 100,00 & 0,00 & - & - \\
\hline Out & 97,50 & 0,00 & 2,50 & 0,00 & 97,50 & 2,50 & - & - \\
\hline Nov & 65,00 & 20,00 & 12,50 & 2,50 & 66,70 & 33,30 & 0,00 & 100,00 \\
\hline Dez & 55,00 & 17,50 & 22,50 & 5,00 & 53,80 & 46,20 & 50,00 & 50,00 \\
\hline
\end{tabular}

Obs.: - significa impossibilidade de cálculo por ocorrência de zero como divisor.

Analisando os valores de $\mathrm{P}(\mathrm{S})$ na Tabela 2 verifica-se que há uma tendência predominante de possíveis períodos secos na maior parte dos meses do ano, sendo os meses de maio a outubro aqueles em que são esperados os maiores números de dias secos. Junho, julho, agosto e setembro são certamente secos, pois apresentaram probabilidade de $100 \%$. Março, por sua vez, foi o mês com menor possibilidade de ocorrência de períodos secos, com probabilidade de 32,5\%.
A Tabela 2 mostra que, entre os meses de maio e outubro, as menores probabilidades de ocorrência de chuvas favorecem a produção e a colheita de uvas finas, uma importante cultura para a geração de empregos e divisas na região de Juazeiro, BA.

\section{Discussão}

Confrontando os valores de precipitações médias mensais obtidas com aquela comumente recomendada $(75 \%)$ para a utilização no 
dimensionamento agronômico de sistemas de irrigação, constatam-se grandes discrepâncias. Com exceção do mês de agosto, todas as precipitações médias apresentaram probabilidade de ocorrência inferior a 50\%; portanto, muito abaixo do nível recomendado para dimensionamento de projetos de irrigação que, segundo Bernardo (2006), é de três em cada quatro anos $(75 \%)$ ou de quatro em cada cinco anos (80\%). Pode-se atribuir esse comportamento à assimetria positiva apresentada pelas precipitações totais do período estudado, realçando a boa aderência das mesmas a distribuição gama. Por esta razão, verifica-se que houve alta frequência de precipitação esperada no período seco e pequena frequência esperada de precipitações no período considerado bastante chuvoso ao longo dos anos em cada mês.

Segundo Moreira et al. (2010) e Pizzato et al. (2012), a utilização da média como parâmetro de dimensionamento provoca subdimensionamento de sistemas de irrigações, ocasionando danos ao produtor. Pinto et al. (2012) destacam que, projetos de dimensionamento de sistemas de irrigação partindo da precipitação média, provavelmente ficarão subdimensionados, podendo não atender às necessidades hídricas da cultura e, com isso, reduzir os rendimentos da mesma. Araújo et al. (2001) enfatizam a importância de estudos probabilísticos, considerando que o uso da chuva média no planejamento de atividades agrícolas é inadequado.

De modo geral, os menores valores de estimativas do parâmetro $\alpha$ ocorreram nos meses com menor índice médio de precipitação. Resultados semelhantes foram encontrados por Moreira et al. (2010) e Pizzato et al. (2012), estudando a distribuição e probabilidade de ocorrência de chuvas no município de Nova Maringá e a distribuição de probabilidade de ocorrência de precipitações em Cáceres, respectivamente, ambos no estado do Mato Grosso.

O mês de agosto apresentou o maior valor para o parâmetro $\alpha$ e o menor valor para o parâmetro $\beta 3,82$ e 1,00 , respectivamente. Tal fato sugere que, neste mês, o regime de precipitação pluvial mostra-se mais homogêneo, uma vez que elevados valores do parâmetro $\alpha$ e baixos valores do parâmetro $\beta$ conferem um menor grau de assimetria em torno do valor de precipitação mais frequente. Isso pode ser confirmado na Tabela 1, por meio das precipitações prováveis para os diferentes níveis de probabilidade do referido mês, apresentando menor amplitude e menor média mensal de precipitação.
Moreira et al. (2010) constataram que os menores valores do parâmetro $\alpha$, coincidiram com os meses em que ocorreram os menores totais de precipitações, enquanto os maiores valores de $\alpha$, ocorreram nos meses com maiores totais de precipitações. Martins et al. (2010) observaram que a variação interanual de precipitação é menos acentuada nos meses em que os valores de $\alpha$ são maiores, podendo este parâmetro ser utilizado na determinação de períodos regulares de precipitação. Blain et al. (2007), estudando a distribuição temporal da precipitação pluvial mensal observada no posto meteorológico do Instituto Agronômico, em Campinas-SP, observaram que, no mês de dezembro, para um dos quatro períodos analisados, ocorreu um regime mais homogêneo de precipitação pluvial, com maior ocorrência de valores próximos à moda e menor grau de assimetria em torno desta, indicados pelo maior valor de $\alpha$ e menor valor de $\beta$.

Analisando os valores de $\mathrm{P}(\mathrm{C})$ no período considerando chuvoso, constatou-se que dezembro, fevereiro e março apresentaram maior probabilidade de ocorrência de meses chuvosos, respectivamente, com $22,5 \%, 27,5 \%$ e $20,0 \%$. O mês de março apresentou-se como aquele com maior probabilidade de ser bastante chuvoso (25,0\%), ou seja, é esperado que, a cada quatro anos, março apresente um volume total de chuvas maior do que $200 \mathrm{~mm}$.

Do ponto de vista das probabilidades condicionais, para o caso de $\mathrm{P}(\mathrm{S} / \mathrm{S})$ e $\mathrm{P}(\mathrm{S} / \mathrm{C})$, verifica-se que o mês de março apresenta-se como aquele com menor probabilidade de ser seco, independentemente de fevereiro ter sido seco $(33,3 \%)$ ou chuvoso $(31,8 \%)$. Nota-se, ainda, que este é o mês que apresenta maior chance de ser chuvoso, caso o mês anterior tenha sido seco $(66,7 \%)$ ou chuvoso $(68,2 \%)$. Portanto, apesar do período chuvoso na região de estudo ser de novembro a março, chuvas acima de $45 \mathrm{~mm}$. mês ${ }^{1}$, com $60 \%$ de probabilidade de ocorrência, são observadas nos meses de fevereiro e março.

A produtividade agrícola é fortemente influenciada pela oferta pluviométrica e pela frequência e duração dos períodos secos (Keller Filho et al., 2006). Favero et al. (2008) concluíram que, em vinhedo não irrigado em Três Corações, MG, a videira Syrah expressou no ciclo de inverno, índices de desenvolvimento, produção e qualidade suficientes para viabilizar a cultura na região, e que a ocorrência de baixos índices de precipitação e amplitude térmica, característica do período de colheita do inverno favoreceram a qualidade da uva. Mota et al. (2010) destacam que a alteração do ciclo de produção de uvas para 
vinho fino, com a colheita acontecendo nos meses mais secos do ano, contribui para o avanço da maturação das bagas e a melhoria da qualidade do mosto, expressa principalmente pelo teor de açúcar, acidez e compostos fenólicos.

É importante destacar que, por menores que sejam as probabilidades de ocorrência de chuvas nos meses de maio a outubro, caso elas aconteçam, podem prejudicar de forma significativa a colheita da uva, cujas bagas tendem a rachar, apodrecer e cair. A ocorrência de chuva, principalmente durante a época de colheita da uva, aumenta a probabilidade de excesso de água danificando os cachos por rachadura das bagas e por podridões (Pedro Júnior et al., 2007). Carvalho et al. (2011) atribuem a podridão de cachos de uva madura ao fungo Glomerella cingulata (Penz.) Penz et Sacc; de acordo com Mandelli (2006), a disseminação desse fungo ocorre por meio de respingos de chuva com vento. Uma alternativa que permite minimizar os danos causados pela ocorrência de chuva na fase de maturação é a antecipação da colheita, diminuindo, porém, a qualidade da uva, devido às bagas não atingirem a maturação completa (Jacskon \& Cherry, 1987).

Santos et al. (2011), estudando os impactos ecofisiológicos no cultivo de variedade de uvas híbridas e finas, conduzidas sob dupla poda, em Jundiaí-SP, verificaram que chuvas mais bem distribuídas e desconcentradas no período do estabelecimento do fruto até a colheita, proporcionaram condições mais favoráveis para a maturação fenólica das bagas.

\section{Conclusão}

Para todos os meses, os dados de precipitação pluviométrica ajustaram bem a distribuição gama, podendo esta ser utilizada para estimar a precipitação pluvial provável em diferentes níveis de probabilidade na região de Juazeiro-BA.

A melhor época para o plantio de sequeiro na região de Juazeiro/BA é o bimestre fevereiro e março, devido ter a menor probabilidade de ocorrência de períodos secos e maior possibilidade de períodos chuvosos consecutivos.

As menores probabilidades de ocorrência de chuva de maio a outubro proporcionam menor risco de perdas na colheita de uvas finas em cultivo irrigado, na região de Juazeiro, BA.

\section{Referências}

ANDRADE, A. R. S. FREITAS, J. C.; BRITO, J. I. B.; GUERRA, H. O. C.; XAVIER, J. F. 2009. Aplicação da probabilidade condicional e do processo de cadeia de Markov na análise da ocorrência de períodos secos e chuvosos para o município de Garanhuns, PE, Brasil. Ambi-Agua, v. 4 , n. 1 , p. $169-182$.

ARAÚJO, W. F.; ANDRADE JÚNIOR, A. S.; MEDEIROS, R. D.; SAMPAIO, R. A. 2001. Precipitação pluviométrica mensal provável em Boa Vista, Estado de Roraima, Brasil. Revista Brasileira de Engenharia Agrícola e Ambiental, v. 5, n. 3, p. 563-567.

ASSIS, F. N.; ARRUDA, H. V.; PEREIRA, A. R. 1996. Aplicações de estatística à climatologia: teoria e prática. Pelotas: Universidade Federal de Pelotas.

ÁVILA, L. F.; MELLO, C. R.; VIOLA, M. R. 2009. Mapeamento da precipitação mínima provável para o sul de Minas Gerais. Revista Brasileira de Engenharia Agrícola e Ambiental, v. 13, p. 906-915. Suplemento.

BALME, M.; VISCHEL, T.; LEBEL, T.; PEUGEOT, C.; GALLE, S. 2006. Assessing the water balance in the Sahel: Impact of small-scale rainfall variability on runoff. Part 1: Rainfall variability analysis. Journal of Hydrology, v. 33, p. 336-348.

BARRETO, H. B. F.; PEREIRA, G. M.; SANTOS, W. O.; FREIRE, S. G. C.; MAIA, P. M. E. 2015. Avaliação de funções de probabilidade e relação-intensidade de duração intensidade de duração - frequência para precipitação extrema em Nepomuceno-MG. Global Science and Technology, v. 8, n. 2, p. 118127.

BERNARDO, S. 2006. Irrigação: Total, suplementar, com déficit e de salvação. Irrigação e Tecnologia Moderna-ITEM, n. 71/72, p. 64-68.

BLAIN, G. C.; PIEDADE, S. M. S.; CAMARGO, M. B. P.; GIAROLLA. A. 2007. Distribuição temporal da precipitação pluvial mensal observada no posto meteorológico do Instituto Agronômico, em Campinas, SP. Bragantia, v. 66, n. 2, p. 347-355.

CARVALHO, M. C.; HAMADA. E.; GARRIDO, L. DA R.; ANGELLOTI, F. 2011. Impactos das mudanças climáticas sobre a distribuição espacial da podridão da uva madura em videira. Anais Congresso Interinstitucional de Iniciação Científica, Campinas: Embrapa Monitoramento por Satélite. 1. CD ROM. 
FAVERO, A. C.; AMORIM, D. A.; MOTA, R. V.; SOARES, A. M.; REGINA, M. A. 2008 Viabilidade de produção da videira 'Syrah', em ciclo de outono inverno, na região sul de Minas Gerais. Revista Brasileira de Fruticultura, v. 30, p. 685-690.

JACSK, O. N., D. I.; CHERRY, N. J. 1987. Prediction of a district's grape - ripening capacity using a latitude-temperature index (LTI). American Journal of Enology and Viticulture, v. 39, p. 19-28.

KELLER FILHO, T.; ZULLO JUNIOR, J.; LIMA, P. R. S. DE R. 2006. Análise da transição entre dias secos e chuvosos por meio da cadeia de Markov de terceira ordem. Pesquisa Agropecuária Brasileira, v. 41, n. 9, p. 1341-1349.

MANDELLI, F. 2006. Comportamento meteorológico e sua influência na vindima de 2006 na Serra Gaúcha. Bento Gonçalves: Embrapa Uva e Vinho.

MARTINS, J. A.; DALLACORT, R.; INOUE, M. H.; SANTI, A.; KOLLING, E. M.; COLETTI, A. J. 2010. Probabilidade de precipitação para a microrregião de Tangará da Serra, Estado do Mato Grosso. Pesquisa Agropecuária Tropical, v. 40, n. 3, p. 291-296.

MOREIRA, P. S. P.; DALLACORT, R.; MAGALHÃES, R. A.; INOUE, M. H.; STIELER, M. C.; SILVA, D. J.; MARTINS, J. A. 2010. Distribuição e probabilidade de ocorrência de chuvas no município de Nova Maringá-MT. Revista de Ciências Agro-Ambientais, v. 8, n. 1, p. 9-20.

MOTA, R.V.; SILVA, C. P. C.; FAVERO, A. C.; PURGATTO, E.; SHIGA, T. M.; REGINA, M. A. 2010. Composição físico-química de uvas para vinho fino em ciclos de verão e inverno. Revista Brasileira de Fruticultura, v. 32, p. 1127-1137.

PEDRO JÚNIOR, M. J.; PEZZOPANE, J. R. M.; HERNANDES, J. L.; LULU, J.; DE CASTRO, J. V. 2007. Avaliações microclimáticas e das características de qualidade da uva de mesa
'Romana' com proteção individual dos cachos. Bragantia, v. 66, n. 1, p. 165-171.

PINTO M. F.; ALVES, D. G.; PAUlinO, J.; COELHO, R. D. 2012. Distribuição de frequência da precipitação e sua aplicação no dimensionamento de projetos de irrigação suplementar. Revista Brasileira de Agricultura Irrigada, v. 6, n. 4, p. 303-313.

PIZZATO, J. A.; DALLACORT, R.; TIEPPO, R. C.; MODOLO, A. J.; CREMON, C.; MOREIRA, P. S. P. 2012. Distribuição e probabilidade de ocorrência de precipitação em Cáceres (MT). Pesquisa Agropecuária Tropical, v. 42, n. 2, p. 137-142.

SANTOS, A. O.; HERNANDES, J. L.; PEDRO JÚNIOR, M. J.; PEREIRA, S. E. 2011. Composição da produção e qualidade da Uva em videira cultivada sob dupla poda e regime microclimático estacional contrastante. Revista Brasileira de Fruticultura, v. 33, n. 4, p. 11351154.

SOUZA, R. R.; ROSA, D. B.; NASCIMENTO, L. A.; LIMA, P. R. M. 2006. Estudo da variabilidade pluviométrica no extremo norte do Estado de Mato Grosso entre os anos de 1990 a 1996. Revista Geoambiente, v. 1, n. 7, p. 89-107.

THOM, H. C. S. 1951. A frequency distribution for precipitation. Bulletin of American Meteorological Society, v. 32, n. 10, p. 117-122.

VELA, R. H. N.; DALLACORT, R.; NIED, A. H. 2007. Distribuição decendial, mensal e totais de precipitação na região de Tangará da Serra-MT. Anais Congresso Brasileiro de Engenharia Agrícola, SBEA, Bonito, pp. 1-4.

VIEIRA, J. P. G.; DE SOUZA, M. J. H.; TEIXEIRA, J. M.; DE CARVALHO, F. P. 2010. Estudo da precipitação mensal durante a estação chuvosa em Diamantina, Minas Gerais. Revista Brasileira de Engenharia Agrícola e Ambiental, v. 14, n. 7, p. 762-767. 